Journal of Mathematics and Statistics Studies (JMSS)

ISSN: 2709-4200

DOI Prefix: 10.32996/jmss

www. al-kindipublisher.com/index.php/jmss

\title{
Stochastic Diffusion Process Based on Generalized Brody Curve: Application to Real Data
}

Ahmed Nafidi ${ }^{1}$, Oussama Rida ${ }^{2 *}$ and Boujemaa Achchab ${ }^{3}$

${ }^{123}$ Hassan First University of Settat, National School of Applied Sciences, Department of Mathematics and Informatics, LAMSAD, B.P. 280, 26100 Berrechid, Morocco.

Corresponding Author: Oussama Rida, E-mail: oussamar@gmail.com

\section{ARTICLE INFO}

Received: November 18, 2020

Accepted: January 10, 2021

Volume: 2

Issue: 1

DOI: 10.32996/jmss.2021.2.1.1

\section{KEYWORDS}

Stochastic differential equation, Generalized Brody curve, Maximum likelihood, Optimization algorithms, Simulation, life expectancy

\section{ABSTRACT}

A new stochastic diffusion process based on Generalized Brody curve is proposed. Such a process can be considered as an extension of the nonhomogeneous lognormal diffusion process. From the corresponding Itô's stochastic differential equation (SDE), firstly we establish the probabilistic characteristics of the studied process, such as the solution to the SDE, the probability transition density function and their distribution, the moments function, in particular the conditional and non-conditional trend functions. Secondly, we treat the parameters estimation problem by using the maximum likelihood method in basis of the discrete sampling, thus we obtain nonlinear equations that can be solved by metaheuristic optimization algorithms such as simulated annealing and variable search neighborhood. Finally, we perform a simulation studies and we apply the model to the data of life expectancy at birth in Morocco.

\section{Introduction}

Life expectancy at birth, commonly used as a measure of overall development of a country, has risen over the last ten years in most of the countries of the world. This has a special significance for the developing world since they are seeking for achieving socio-economic progress through investing extensively on social sectors like health, education, sanitation, environmental management and sustainability, and social safety nets. Per capita income of the developing countries has increased and converted into increased level of expenditure on medical treatment and composite commodities. Enhancements in poverty rate, nutrition, adult literacy, access to safe drinking water, chronic diseases, and sanitation have also been impressive over the years that would have a positive impact on life expectancy. Nevertheless, in serval countries of the developing world, SubSaharan Africa in particular, life expectancy has been decreasing. In some of the countries although income and health expenditure is increasing, life expectancy is decreasing, see Kabir (2008).

The first paper associated to this theory on stochastic modeling of the health state of an individual was published by Janssen \& Skiadas (1995). An application on the Belgium and France data was presented by the authors in the Royal Association of Belgian Actuaries in a meeting in 1995 celebrating the 100 years of the Association. The modeling approach was based on finding the distribution of the first exit time of a diffusion process expressing the health state of a person from a barrier. The related theory can be found in Janssen \& Skiadas (1995), Skiadas \& Skiadas (2007), Skiadas \& Skiadas (2010), Skiadas \& Skiadas (2011) lately in the International Encyclopedia of Statistical Science, Lovric (2011) and Skiadas (2011). The publications mentionned before are focused on designing and implementing of a first exit time model for mortality including the infant mortality.

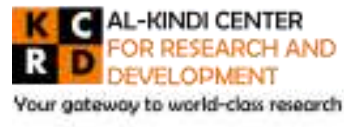

Published by Al-KindiCenter for Research and Development. Copyright (c) the author(s). This is an open access article under CC BY license (https://creativecommons.org/licenses/by/4.0/) 
The proposed model is the stochastic process of diffusion based on generalized brody curve (Brody \& Lardy (1946), Brown \& Butts (1972)) given by :

$$
y(t)=b_{0}\left(1-a e^{-b t}\right)^{p}
$$

This model is an extension of a non-homogeneous lognormal diffusion process, see (Gutiérrez et al. (1999), Nafidi et al. (2019), Nafidi et al. (2020)) and can be defined from the perspective of Kolmogrov partial differential equations and stochastic differential equation SDE.

The mean function of the process is a curve, whose bound may depend on the initial value of the variable under analysis. The predictive potential is one of the strong points of these dynamic models. In order to accurately apply this model, we need reliable estimates of the parameters addressed. In our situation, we use maximum likelihood to tackle the problem of estimation. Thus, a complex system of equations arises, whose solution can not be ensured by classical numerical methods. To overcome this problem, we propose the use of of metaheuristic optimization algorithms such as simulated annealing (SA) and variable neighborhood search (VNS).

In this article, we explore the possibilities of using a new stochatic diffusion process based on Generalized Brody curve to model Life expectancy at birth, total (years) - Morocco. In the second section, we define this process as a solution of Itô's stochastic differential equation (SDE) and then, using Itô's formula, the analytical expression of this process is found, after which the trend and conditional trend functions are determined. In Section 3, the parameter estimators of the proposed process are calculated by optimization methods as a hybrid combination, firstly, the simulated annealing used as local search for the variable neighborhood search to estimate the parameters from the log-likelihood equation. Therefore, a simulation study is performed to illustrate the model. In the last section, the model is applied to time-serie data of Life expectancy at birth, total (years) in Morocco and provides sufficiently good results in term of forcasting accuaracy.

\section{The proposed model and probabilistic characteristics}

\subsection{Model and assumptions}

The proposed model is a one dimensional diffusion process with values in $(0, \infty)$ and is defined by the process $\left\{x(t): t \in\left[t_{0}, T\right]\right\}$ solution of the following non linear stochastic differential equation (SDE):

$$
d x(t)=\frac{p a b}{e^{b t}-a} x(t) d t+\sigma x(t) d w(t), x\left(t_{0}\right)=x_{t_{0}}
$$

Where $a, b, p, \sigma>0$ are real parameters and $w(t)$ is a one-dimensional standard Wiener process and $x_{t_{0}}>0$ is almost surely a constant.

by assuming the following conditions:

- $\quad \lim _{h \rightarrow 0} \frac{1}{h} \int_{|y-x|>\epsilon} P(d y, t+h \mid x, t)=0$

- $\lim _{h \rightarrow 0} \frac{1}{h} \int_{|y-x| \leq \epsilon}(y-x) P(d y, t+h \mid x, t)=A_{1}(t, x)=\frac{p a b}{e^{b t}-a} x$

- $\quad \lim _{h \rightarrow 0} \frac{1}{h} \int_{|y-x| \leq \epsilon}(y-x)^{2} P(d y, t+h \mid x, t)=A_{2}(t, x)=\sigma^{2} x^{2}>0$

- $\quad$ the higher-order infinitesimal moments are null ;

Alternatively the proposed model can be defined by kolmogrov backward and forward, equations given by :

$$
\begin{gathered}
\frac{\partial f}{\partial s}+\frac{p a b}{e^{b t}-a} x \frac{\partial f}{\partial x}+\frac{\sigma^{2}}{2} x^{2} \frac{\partial^{2} f^{2}}{\partial x^{2}}=0 \\
-\frac{\partial f}{\partial t}-\frac{p a b}{e^{b t}-a} \frac{\partial(y f)}{\partial y}+\frac{\sigma^{2}}{2} \frac{\partial^{2}\left(y^{2} f\right)}{\partial y^{2}}=0
\end{gathered}
$$




\subsection{Analytical expression of the proposed model}

We describe the main characteristics of the process, especially for forecasting purposes. These characteristics are the mean function (which by the structure of the model is a Generalized Brody curve, and thus particularly suitable for fitting and predicting).

From the SDE Eq.(2) we proceed the transformation $y(t)=\log (x(t))$ and apply the Itô formula, then we have the analytical expression :

$$
y(t)=y_{0}+p \log \left(\frac{1-a e^{-b t}}{1-a e^{-b t_{0}}}\right)-\frac{\sigma^{2}}{2}\left(t-t_{0}\right)+\sigma\left(w(t)-w\left(t_{0}\right)\right)
$$

By substituting, we have the following expression:

$$
x(t)=x_{0}\left(\frac{1-a e^{-b t}}{1-a e^{-b t_{0}}}\right)^{p} \exp \left(\sigma\left(w(t)-w\left(t_{0}\right)\right)-\frac{\sigma^{2}}{2}\left(t-t_{0}\right)\right), t \geq 0
$$

\subsection{Distribution of the process}

Since for $\mathrm{s}<\mathrm{t}$, the variable $\mathrm{y}(\mathrm{t}) \mid \mathrm{y}(\mathrm{s})=\mathrm{y}_{\mathrm{s}} \sim \mathcal{N}\left[\mathrm{g}\left(\mathrm{s}, \mathrm{t}, \mathrm{x}_{\mathrm{s}}\right), \sigma^{2}(\mathrm{t}-\mathrm{s})\right]$, consiquently, $\mathrm{x}(\mathrm{t}) \mid \mathrm{x}(\mathrm{s})=\mathrm{x}_{\mathrm{s}} \sim \Lambda\left[\mathrm{g}\left(\mathrm{s}, \mathrm{t}, \mathrm{x}_{\mathrm{s}}\right), \sigma^{2}(\mathrm{t}-\right.$ $\mathrm{s})$ ] with $\mathrm{g}\left(\mathrm{s}, \mathrm{t}, \mathrm{x}_{\mathrm{s}}\right)=\log \left(\mathrm{x}_{\mathrm{s}}\right)+\operatorname{plog}\left(\frac{1-\mathrm{a} \mathrm{e}^{-\mathrm{bt}}}{1-\mathrm{a} \mathrm{e}^{-\mathrm{bs}}}\right)-\frac{\sigma^{2}}{2}(\mathrm{t}-\mathrm{s})$. Then, the transition probability density function (TPDF) of the process is :

$$
f(x, t \mid y, s)=\frac{1}{x \sqrt{2 \pi \sigma^{2}(t-s)}} \exp \left(-\frac{\left[\log \left(\frac{x}{y}\right)-p \log \left(\frac{1-a e^{-b t}}{1-a e^{-b s}}\right)+\frac{\sigma^{2}}{2}(t-s)\right]^{2}}{2 \sigma^{2}(t-s)}\right)
$$

which corresponds to a lognormal distribution, that is :

$$
x(t) \mid x(s)=y \sim \wedge\left[\log (y)+p \log \left(\frac{1-a e^{-b t}}{1-a e^{-b s}}\right)-\frac{\sigma^{2}}{2}(t-s), \sigma^{2}(t-s)\right]
$$

\subsection{Moments of the process}

By using the propreties of the lognormal distribution, that the $r$-th conditional moment of the process is expressed by :

$$
\begin{array}{cc}
\mathbb{E}\left[x^{r}(t) \mid x(s)=x_{s}\right]= & \exp \left(r g\left(s, t, x_{s}\right)+\frac{r^{2} \sigma^{2}}{2}(t-s)\right) \\
= & x_{s}^{r}\left(\frac{1-a e^{-b t}}{1-a e^{-b s}}\right)^{p r} e^{-\frac{r}{2}(1-r) \sigma^{2}(t-s)} .
\end{array}
$$

In short, by considering the case $r=1$, the conditional trend function (CTF) of the process is given by:

$$
\mathbb{E}\left[x(t) \mid x(s)=x_{s}\right]=x_{s}\left(\frac{1-a e^{-b t}}{1-a e^{-b t_{0}}}\right)^{P}, t>s
$$

In addition, taking into account the initial condition $P\left[x\left(t_{0}\right)=x_{0}\right]=1$, the trend function (TF) of the process is given by:

$$
\mathbb{E}[x(t)]=x_{0}\left(\frac{1-a e^{-b t}}{1-a e^{-b t_{0}}}\right)^{P}, t>t_{0}
$$


- In absence of white noise (i.e. $\sigma=0$ ), by a simple integration, the solution of the ODE associated to the SDE Eq.(2) is $x(t)=k\left(1-a e^{-b t}\right)^{p}$, which is proportional to generalized Brody curve curve Eq.(1) .

- $\quad$ Otherwise, the TF given in Eq.(6) is proportional to generalized Goel-Okumoto curve curve. In addition we can obtain the quantile function where $z_{\alpha}$ is the $\alpha$-quantile of a standard normal.

$$
P_{\alpha}(t)=x_{0}\left(\frac{1-a e^{-b t}}{1-a e^{-b t_{0}}}\right)^{P} \exp \left\{-\frac{\sigma^{2}}{2}\left(t-t_{0}\right)+z_{\alpha} \sigma \sqrt{t-t_{0}}\right\}
$$

\section{. Estimation of parameters}

Since we have the explicit expression of the tpdf, we can estimate the parameters $p, b, a$ and $\sigma^{2}$ by employing the maximum likelihood method. Let us consider a discrete sampling of the process $x_{t_{1}}, x_{t_{2}}, \ldots, x_{t_{n}}$ at the instants $t_{1}, t_{2}, \ldots, t_{n}$. In addition, we assume that $t_{i}-t_{i-1}=h$, for $i=2, \ldots, n$ with $h$ a constant. We use the abbreviation $x_{t_{i}}=x_{i}$ Then, by assuming the initial condition $\mathbb{P}\left[x\left(t_{1}\right)=x_{1}\right]=1$, the associated likelihood function is obtained, from Eq.(4), as follow:

$$
\begin{gathered}
\mathbb{L}\left(x_{1}, x_{2}, \ldots, x_{n} ; a, b, p, \sigma^{2}\right)=\prod_{i=2}^{n} f\left(x_{i}, t_{i} \mid x_{i-1}, t_{i-1}\right) . \\
\mathbb{L}\left(a, b, p, \sigma^{2}\right)=\prod_{i=2}^{n} f\left(x_{i}, t_{i} \mid x_{i}-1, t_{i}-1\right)=\frac{1}{x_{j} \sqrt{2 \pi \sigma^{2}\left(t_{i}-t_{i-1}\right)}} \\
\times \exp \left(-\frac{1}{2} \frac{\left[\log \left(\frac{x_{i}}{x_{i-1}}\right)-p \log \left(\frac{1-a e^{-b t_{i}}}{1-a e^{-b_{i}-1}}\right)+\frac{\sigma^{2}}{2}\left(t_{i}-t_{i-1}\right)\right]^{2}}{\left(t_{i}-t_{i-1}\right) \sigma^{2}}\right)
\end{gathered}
$$

To simplify the computational aspect of the above function, we use the log-likelihood function:

$$
\begin{gathered}
\log \mathbb{L}\left(x_{1}, x_{2}, \ldots, x_{n} ; a, b, p, \sigma^{2}\right)=-\frac{n-1}{2} \log (2 \pi h)-\frac{n-1}{2} \log \sigma^{2}-\sum_{i=2}^{n} \log x_{i} \\
-\frac{1}{2 h \sigma^{2}} \sum_{i=2}^{n}\left[\log \left(\frac{x_{i}}{x_{i-1}}\right)-p \log \left(\frac{1-a e^{-b t_{i}}}{1-a e^{-b t_{i-1}}}\right)+\frac{\sigma^{2}}{2} h\right]^{2}
\end{gathered}
$$

By differentiating the log-likelihood function with respect to $p, b, a$ and $\sigma^{2}$ with $t_{i}-t_{i-1}$ and applying the maximum likelihood principe, we have :

$$
\begin{aligned}
\frac{\partial \log \left(\mathbb{L}\left(a, b, p, \sigma^{2}\right)\right)}{\partial p}= & \sum_{i=2}^{n} \frac{\log \left(\frac{x_{i}}{x_{i-1}}\right)-p \log \left(\frac{1-a e^{-b t_{i}}}{1-a e^{-b t_{i-1}}}\right)+\frac{\sigma^{2}}{2} h}{h} \\
& \times \log \left(\frac{1-a e^{-b t_{i}}}{1-a e^{-b t_{i-1}}}\right)=0
\end{aligned}
$$




$$
\begin{aligned}
& \frac{\partial \log \left(\mathbb{L}\left(a, b, p, \sigma^{2}\right)\right)}{\partial b}=\sum_{i=2}^{n} \frac{\log \left(\frac{x_{i}}{x_{i-1}}\right)-p \log \left(\frac{1-a e^{-b t_{i}}}{1-a e^{-b t_{i-1}}}\right)+\frac{\sigma^{2}}{2} h}{h} \\
& \times \frac{t_{i-1} e^{-b t_{i-1}-1}\left(1-a e^{-b t_{i}}\right)-t_{i} e^{-b t_{i}-1}\left(1-a e^{-b t_{i-1}}\right)}{\left(1-a e^{-b t_{i}}\right)\left(1-a e^{-b t_{i-1}}\right)}=0 \\
& \frac{\partial \log \left(\mathbb{L}\left(a, b, p, \sigma^{2}\right)\right)}{\partial a}=\sum_{i=2}^{n} \frac{\log \left(\frac{x_{i}}{x_{i-1}}\right)-p \log \left(\frac{1-a e^{-b t_{i}}}{1-a e^{-b t_{i-1}}}\right)+\frac{\sigma^{2}}{2} h}{h} \\
& \times \frac{e^{-b t_{i-1}-1}\left(e^{-b t_{i}}\right)}{\left(1-a e^{-b t_{i}}\right)\left(1-a e^{-b t_{i-1}}\right)}=0 \\
& \frac{\partial \log \left(\mathbb{L}\left(a, b, p, \sigma^{2}\right)\right)}{\partial \sigma^{2}}=\sigma^{4} h^{2}(n-1)+4 \sigma^{2} h(n-1)-4 \sum_{i=2}^{n} \log ^{2}\left(\frac{x_{i}}{x_{i-1}}\right) \\
& -4 p^{2} \sum_{i=2}^{n}\left(\log \left(\frac{1-a e^{-b t_{i}}}{1-a e^{-b t_{i-1}}}\right)\right)^{2}+8 p \sum_{i=2}^{n} \log \left(\frac{1-a e^{-b t_{i}}}{1-a e^{-b t_{i-1}}}\right) \log \left(\frac{x_{i}}{x_{i-1}}\right)=0
\end{aligned}
$$

The estimation of the parameters requires the solution to a nonlinear equations, which can be difficult to achieve in this case, inthe other hand the likelihood equations obtained do not have an explicit solution.

To overcome this problem, we suggest two methods :

Simulated annealing (SA) method, and also Variable neighborhood search (VNS), which is increasingly being adopted.

In Our case we will estimate the parameters from the log-likelihood equation defined bellow by the VNS method:

$$
S\left(a, b, p, \sigma^{2}\right)=-\frac{n-1}{2} \log \sigma^{2}-\frac{1}{2 h \sigma^{2}} \sum_{i=2}^{n}\left[\log \left(\frac{x_{i}}{x_{i-1}}\right)-p \log \left(\frac{1-a e^{-b t}}{1-a e^{-b t_{0}}}\right)+\frac{\sigma^{2}}{2} h\right]^{2} .
$$

\subsection{Aspects on optimization methods}

As previously mentioned, an alternative to solving these systems of equations would be the use of stochastic optimization procedures, such as Simulated Annealing (SA) or Variable Neighborhood Search (VNS). These algorithms are built to solve problems of the type $\min f(w)$, with $w \in \Omega$, and in most cases are strongly recommended than classical numerical methods, since they impose less restrictions on the solution space and on the analytical properties of the objective function, In the context of maximum likelihood estimation for distributions, it has been used in works like those of Vera \& Díaz-García (2008) and Román-Román \& Torres-Ruiz (2015).

\subsubsection{Simulated Annealing (SA) Algorithm}

It is a metaheuristic local search algorithm introduced by Kirkpatrick et al. (1983)., inspired by the annealing metallurgical process studied in mechanical statistics. In general the algorithm follows this procedure:

Given a solution $\theta$ for an iteration and $f(\theta)$ the value of the target function, in the next iteration we select a new value $\theta^{\prime}$ in an environment $N_{\theta}$ of $\theta$, and we evaluate the increase of the objective function $\Delta=f\left(\theta^{\prime}\right)-f(\theta)$. if $\Delta \leq 0$, then $\theta^{\prime}$ is selected as the new solution. Otherwise it could probably be accepted $p=\exp (-\Delta / \mathrm{T})$ with $T$ called temperature. Thus, an internal 
loop generates a Markov chain that will be as long as the number of loop iterations. At the end of the loop, the temperature gradually drops and a new Markov chain is generated. At first, the cooling process enables you to select solutions that worsen the objective function (at high temperatures), but as the temperature decreases this type of solutions are no longer tolerated. Consequently, the general application of the algorithm requires:

- Selection of initial algorithm parameters (initial solution $\theta_{0}$, initial temperature $T_{0}$, final temperature, number of iterations of the algorithm (length of the Markov chain, L) and cooling procedure), and a stopping condition.

- Application of the selection procedure for a new solution $L$ times.

- Verification of the stop condition. If not verified, decrease the temperature and return to the previous step.

\subsubsection{Variable Neighborhood Search Algorithm (VNS)}

The basic concept of this algorithm, introduced by Mladenovi, \& Hansen (1997)., is to explore multiple environments in the solution space when a local optimum is found through a local search method. The algorithm is applied in two distinct steps:

The first is to determine a structure of environments in the solution space, $N_{k}, k=1, \ldots, k_{\max }$ and choose an initial solution $\theta_{0}$

The second phase we use a local search method to determine a new solution $\theta^{*}$ in $N_{k}\left(\theta_{0}\right)$. if $\theta^{*}$ causes an improvement in the objective function, then $\theta_{0}=\theta^{*}$ and the search is resumed from $N_{1}\left(\theta_{0}\right)$. In any other case the search continues with the following solution space $N_{k+1}\left(\theta_{0}\right)$.

The described procedure changes the solution spaces each time an improvement is carried out in the objective function. Thus there are variations of this algorithm, to adapt to the different ways in which the structure of environments can change when a local optimum has been attained, as well as various local search methods. In most applications of the Thesis we will use VND (Basic Variable Neighborhood Descent) and its application must take into account the structure of the environment and the local search method.

\subsection{Estimated TF and Estimated CTF}

From Zenha's theorem in Zehna (1966), by replacing the parameters by their estimators in Eq.(5) and Eq.(6), the estimated conditional trend (ECTF) function can be obtained from:

$$
\hat{E}\left[x(t) \mid x(s)=x_{s}\right]=x_{s}\left(\frac{1-\hat{a} e^{-\hat{b t}}}{1-\hat{a} e^{-\hat{b s}}}\right)^{\hat{p}}
$$

and the estimated trend function (ETF) is given by:

$$
\hat{E}[x(t)]=x_{t_{0}}\left(\frac{1-\hat{a} e^{-\hat{b t}}}{1-\hat{a} e^{-\hat{b t} t_{0}}}\right)^{\hat{p}}
$$

Furthermore, the estimated quantile is obtained by replacing the parameters by their estimators in Eq.(7).

$$
\hat{P}_{\alpha}(t)=x_{0}\left(\frac{1-\hat{a} e^{-\hat{b t} t}}{1-\hat{a} e^{-\hat{b} t_{0}}}\right)^{\hat{p}} \exp \left\{-\frac{\hat{\sigma^{2}}}{2}\left(t-t_{0}\right)+z_{\alpha} \hat{\sigma} \sqrt{t-t_{0}}\right\}
$$

\section{Simulation}


We use the algorithm derived from the numerical solution of the (SDE) associated with the process, see Kloeden \& Platen (2013), we simulate 20 trajectories of the process Eq.(3), where we consider 100 observations for every trajectory and from the following discretizing time interval $\left[t_{1}, T\right]: t_{i}=t_{1}+(i-1) h$, for $i=2, \ldots, N$ where $N$ is an integer and $h=\frac{T-t_{1}}{N}>0$ is the discretization step. Figure 1 shows the process with the initial values : $x_{0}=5, t_{0}=0, a=0.6, b=0.2, p=2$ and for each $\sigma$.

Sample paths, $\sigma=0.01$

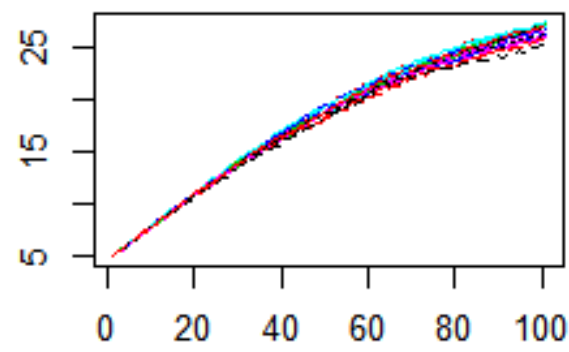

time

\section{Sample paths, $\sigma=0.05$}

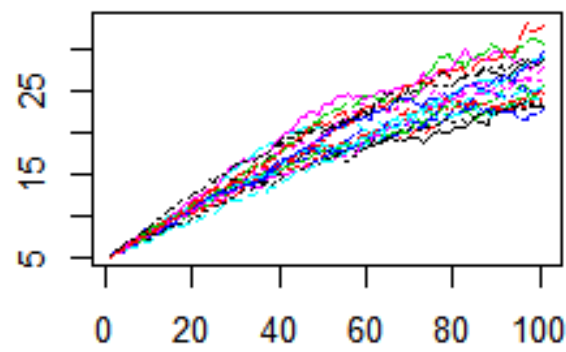

time
Sample paths, $\sigma=0.02$

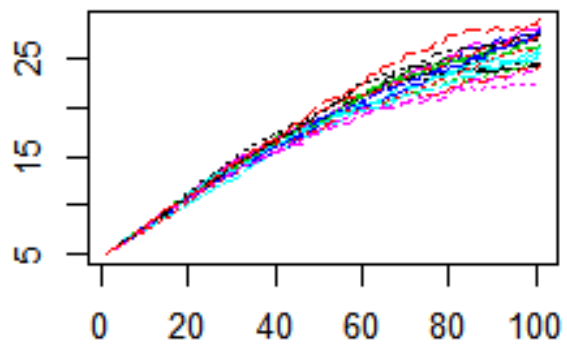

time

\section{Sample paths, $\sigma=0.1$}

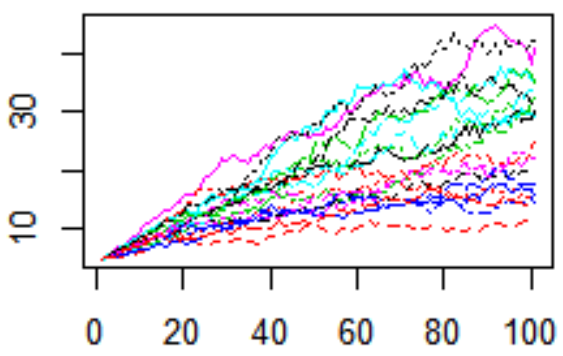

time

Figure 1: Simulated sample path of Generalized Brody process and TF

\section{Application To real data}

The proposed model is applied to Life expectancy at birth, total (years) - Morocco from 1999 to 2018.

Life expectancy at birth represents the number of years a newborn child would live if existing patterns of mortality at the time of its birth were to remainthe same throughout its life.

Mortality rates for different age groups (infants, children, and adults) and overall mortality indicators (life expectancy at birth or survival to a given age) are important indicators of health status in a country. Because data on the occurence and prevalence of diseases are frequently unavailable, mortality rates are often used to classify vulnerable populations. And they are among the indicators most widely used to measure socioeconomic development across countries, see Bilas et al. (2014).

Life expectancy at birth is calculated in a life span table which gives a snapshot of a population's mortality rate at a given time. It does not consequently represent the mortality pattern that a person actually encounters during his / her life, which can be measured in a cohort life table. High mortality in young age groups greatly decrease the life expectancy at birth. But if a person survives his/her childhood of high mortality, he/she may live much longer. For example, in a population with a life expectancy 
at birth of 50, there may be few people dying at age 50. Due to high childhood mortality, life expectancy at birth may be low so that if a person survives his / her childhood, he / she may live longer than 50 years, see, Toson \& Baker (2003).

The data (see Table 1) were provided in World Bank's database². The method employed is composed of two phases:

- Data from 1999-2016 are used to estimate the process parameters by Variable neighborhood search method and by using the $\mathrm{R}$ program, the following estimator values are obtained: $\hat{a}=0.88, \hat{b}=0.219, \hat{p}=0.09$ and $\hat{\sigma}^{2}=0.025$

- Data from 2017-2018 are explored to forecast the expected values of the process. The results in Table 1 resume the behaviour of the conditional and the non-conditional trend functions.

The performance of the process for the short-term forcast with is represented in Figure 2 and Figure 3.

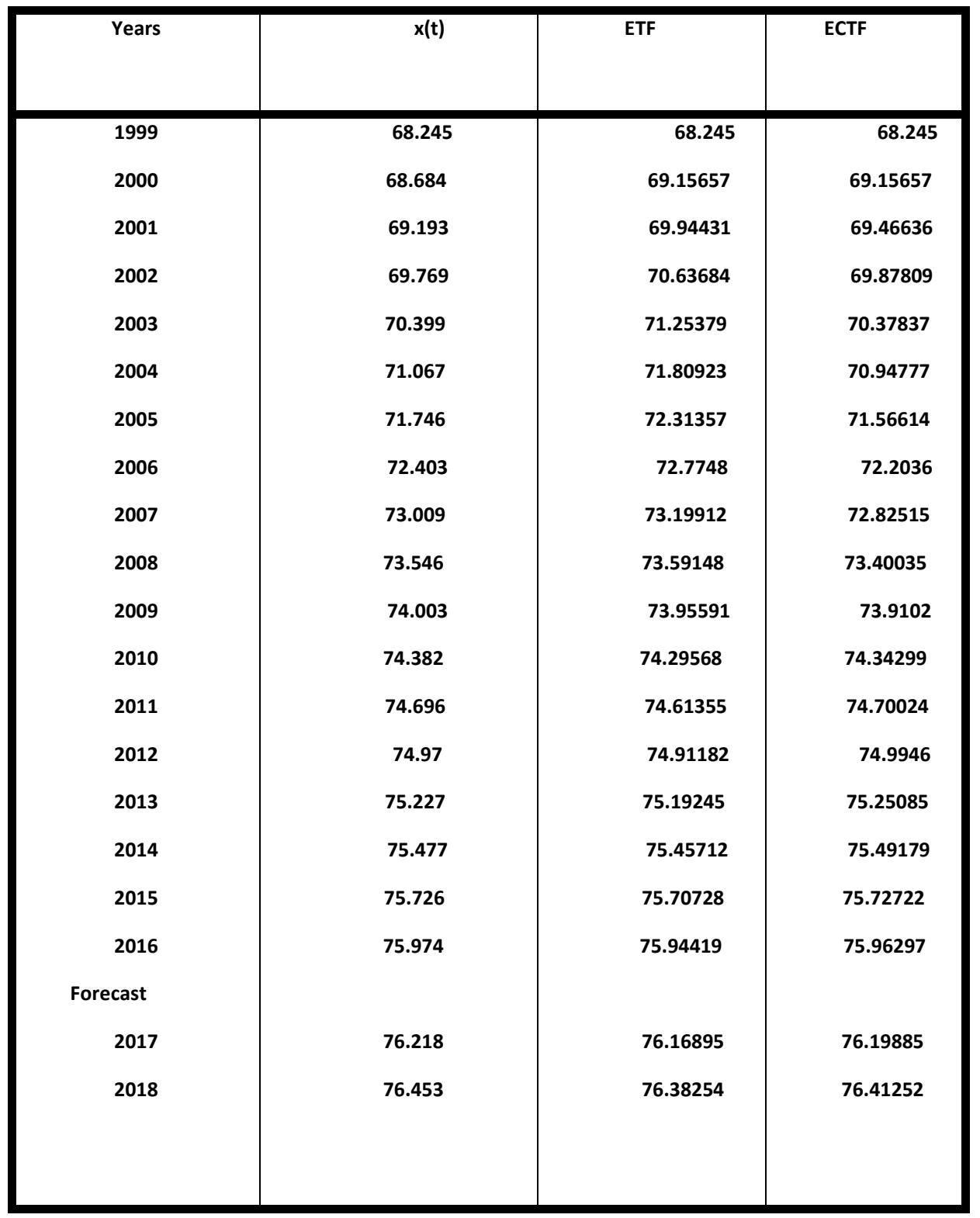

\footnotetext{
${ }^{2}$ https://data.worldbank.org. World bank.life expectancy at birth, total (years) - morocco. 2019.
} 
Table 1 : Life expectancy at birth, ETF and ECTF

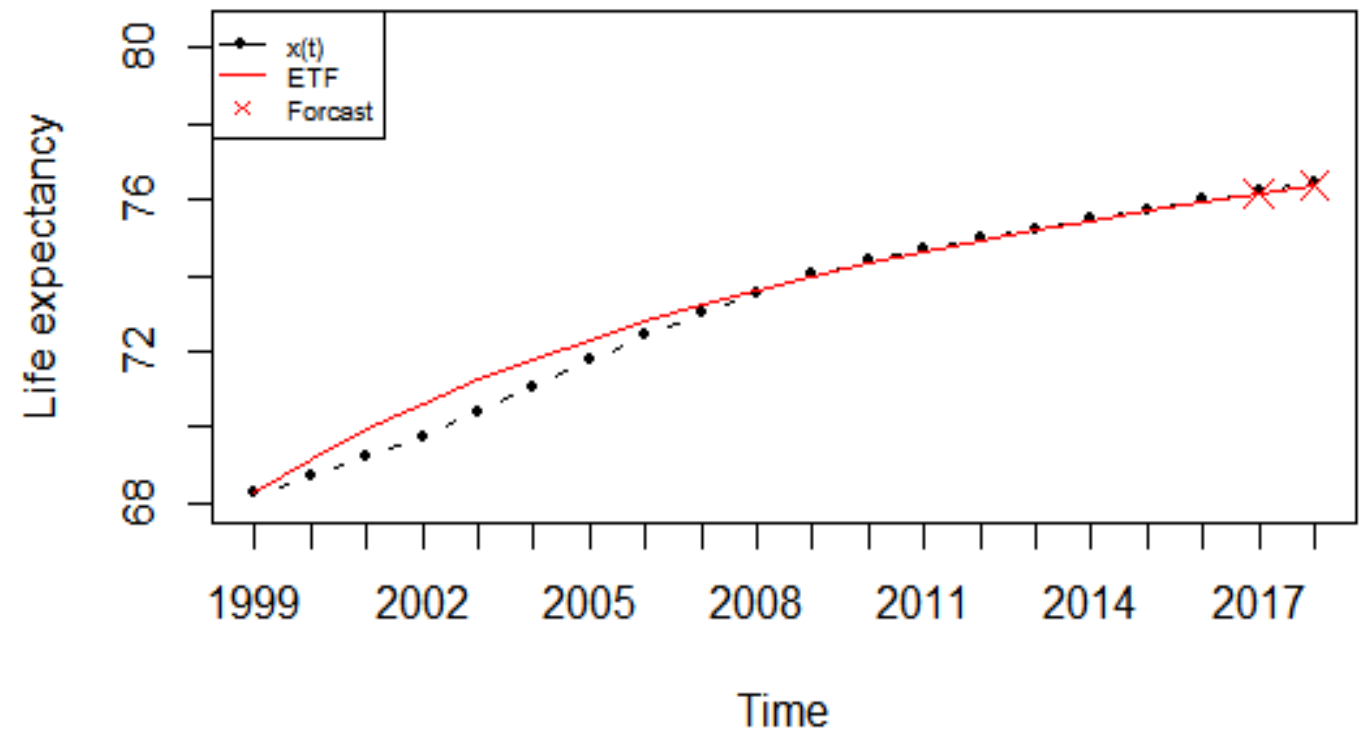

Figure 2 : Observed Data and Estimated TF with forcast (2017-2018)

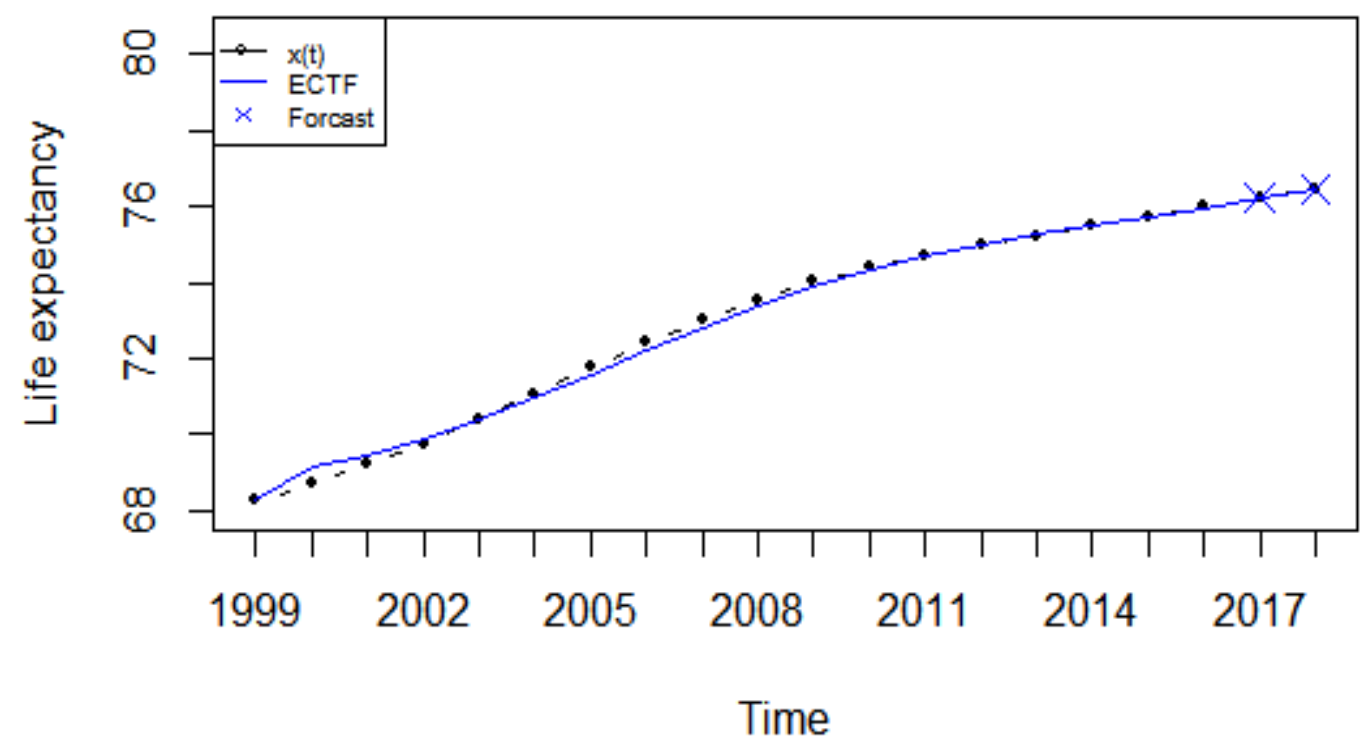

Figure 3 : Observed Data and Estimated CTF with forcast (2017-2018) 
The values fitted with forcasts in accordance with the ETF are slightly better than ECTF, and to illustrate the performance of the model, the mean absolute percentage error (MAPE) and Symmetric mean absolute percentage error (SMAPE) are given by :

$$
M A P E=\frac{1}{N} \sum_{S M A P E}^{N} \frac{1}{N} \sum_{i=1}^{N} \frac{\left|x\left(t_{i}\right)-\hat{x}\left(t_{i}\right)\right|}{x\left(t_{i}\right)} * 100
$$

The accuracy of the forecast can be judged from the MAPE and SMAPE results respectively $0.3 \%$ and $0.3 \%$, which is less than $10 \%$, and so the forecast is highly accurate, see Goodwin \& Lawton (1999).

\section{Conclusion}

In this study of the new Stochastic diffusion process based on Genaralized Brody curve, From a theoretical point of view, we conclude that this process which is of a non-homogeneous nature, is such that we can explicitly establish its probability transition density function in terms of a log-normal distribution in section 2.3 together with its moment functions, and in particular its trend functions in section 2.4. We can also establish parameter estimation results using the maximum likelihood method and optimization methods namely simulated annealing and variable neighborhood search, on the basis of discrete sampling. therefore, the diffusion process based on Genaralized Brody curve we described is followed by a set of statistical results that enable it to be applied to real data.

The proposed model was applied to analyse the Life expectancy at birth in Morocco . This obtained an improved description of the time series considered (1999-2016) and improved short-term forecasts (2017-2018). From the results obtained (see Table 1, Figure 2 and Figure 3), we conclude that when the real case considered is modeled by the diffusion process based on Generalized Brody curve according to the estimation methodology described in Section 3, the fit and prediction achieved, based on ETF and ECTF, present a high degree of accuracy following the the mean absolute percentage error (MAPE) and Symmetric mean absolute percentage error (SMAPE) measures.

For further research and to enhance the capacity of the model, it would be interesting to define a stochastic Generalized brody diffusion process with exogenous factors into the drift, as has been done in the case of the Gompertz process with exogenous factors. In this respect, see Gutiérrez et al. (2006) This would enable us to study the factors affecting the evolution of the life expectancy of Morocco for example: GDP growth, human capital index and labor force.

Acknowledgments: The authors are very grateful to the Editor and referees.

Conflicts of Interest: The authors declare no conflict of interest.

\section{References}

[1] Bilas, V., Franc, S., \& Bošnjak, M. (2014). Determinant factors of life expectancy at birth in the European Union countries. Collegium antropologicum, 38(1), 1-9.

[2] Brody, S., \& Lardy, H. A. (1946). Bioenergetics and growth. The Journal of Physical Chemistry, 50(2), 168-169.

[3] Brown, J., Brown, C. J., \& Butts, W. (1972). A discussion of the genetic aspects of weight, mature weight and rate of maturing in Hereford and Angus cattle. Journal of Animal Science, 34(4), 525-537.

[4] Goodwin, P., \& Lawton, R. (1999). On the asymmetry of the symmetric MAPE. International journal of forecasting, 15(4), 405-408.

[5] Gutiérrez, R., Gutiérrez-Sánchez, R., \& Nafidi, A. (2006). Electricity consumption in Morocco: stochastic Gompertz diffusion analysis with exogenous factors. Applied Energy, 83(10), 1139-1151.

[6] Gutiérrez, R., Román, P., \& Torres, F. (1999). Inference and first-passage-times for the lognormal diffusion process with exogenous factors: application to modelling in economics. Applied Stochastic Models in Business and Industry, 15(4), 325-332.

[7] Janssen, J., \& Skiadas, C. H. (1995). Dynamic modelling of life table data. Applied Stochastic Models and Data Analysis, 11(1), 35-49.

[8] Kabir, M. (2008). Determinants of life expectancy in developing countries. The journal of Developing areas, $185-204$.

[9] Kirkpatrick, S., Gelatt, C. D., \& Vecchi, M. P. (1983). Optimization by simulated annealing. science, 220(4598), 671-680.

[10] Kloeden, P. E., \& Platen, E. (2013). Numerical solution of stochastic differential equations (Vol. 23). Springer Science \& Business Media. 
[11] Lovric, M. (2011). International Encyclopedia of Statistical Science. Springer.

[12] Nafidi, A., Bahij, M., Achchab, B., \& Gutiérrez-Sánchez, R. (2019). The stochastic Weibull diffusion process: Computational aspects and simulation. Applied Mathematics and Computation, 348, 575-587.

[13] Nafidi, A., Bahij, M., Gutiérrez-Sánchez, R., \& Achchab, B. (2020). Two-Parameter Stochastic Weibull Diffusion Model: Statistical Inference and Application to Real Modeling Example. Mathematics, 8(2), 160.

[14] Mladenović, N., \& Hansen, P. (1997). Variable neighborhood search. Computers \& operations research, 24(11), 1097-1100.

[15] Skiadas, C. H. (2011). Recent advances in stochastic modeling. International encyclopedia of statistical science, 1524-1526.

[16] Skiadas, C. H., \& Skiadas, C. (2007). A modeling approach to life table data sets. In Recent advances in stochastic modeling and data analysis (pp. 350-359).

[17] Skiadas, C., \& Skiadas, C. H. (2010). Development, simulation, and application of first-exit-time densities to life table data. Communications in Statistics-Theory and Methods, 39(3), 444-451.

[18] Skiadas, C. H., \& Skiadas, C. (2011). Exploring life expectancy limits: First exit time modeling, parameter analysis and forecasts. In Chaos theory: Modeling, simulation and applications (pp. 357-368).

[19] Toson, B., \& Baker, A. (2003). Life expectancy at birth: methodological options for small populations. London: Office for National Statistics.

[20] Vera, J. F., \& Díaz-García, J. A. (2008). A global simulated annealing heuristic for the three-parameter lognormal maximum likelihood estimation. Computational statistics \& data analysis, 52(12), 5055-5065.

[21] Zehna, P. W. (1966). Invariance of maximum likelihood estimators. Annals of Mathematical Statistics, $37(3), 744$. 